\title{
The effect of oxidation and reduction processes of graphite on physicochemical properties of graphite oxide and reduced graphene oxide
}

\author{
S. Drewniak, ${ }^{* 1}$, T. Pustelny ${ }^{1}$, R. Muzyka ${ }^{2}$, G. Konieczny ${ }^{1}$, P. Kałużyński ${ }^{1}$ \\ ${ }^{I}$ Department of Optoelectronics, Silesian University of Technology, 2A Akademicka St., 44-100 Gliwice, Poland \\ ${ }^{2}$ Institute for Chemical Processing of Coal; 1 Zamkowa St., 41-803 Zabrze, Poland
}

Received December 15, 2014; accepted December 30, 2014; published December 31, 2014

\begin{abstract}
Due to the sensitivity of their physical properties to selected gases, graphite oxide (GrO) and reduced graphene oxide (RGO) can be used in optical gas sensors. The sensor properties of GrO and RGO depend significantly on the technology used for their production. In this paper the effects of oxidation and reduction processes on graphite physicochemical properties were presented.
\end{abstract}

In many production processes, but also in environmental monitoring, an important problem is the detection of selected gases with adequate selectivity and sensitivity. Recently, much attention has been paid to the development of optical gas sensors, acting both on the basis of optical absorption and the phenomenon of propagation of modes in optical planar structures. It is important that the detection of selected gases should be unambiguous and at a low level of their concentration. Attention should also be paid to the response time of sensors (time of gas detection), which must be as short as possible. The metrological properties of optical gas sensor structures depend mainly on gas sensing layers. In the literature concerning gaseous sensors much attention is devoted to sensors based on graphene, graphite oxide (GrO) and reduced graphene oxide (RGO) [1-3]. There are many different methods of production [4-8] graphene based materials, which undoubtedly affects their structural properties. Our earlier studies [9-10] have shown that the sensor properties of graphene based materials significantly depend on the technology used for their production. In the present paper, the effects were investigated of oxidation and reduction processes on graphite physicochemical properties.

The research work presented in this paper was designed to describe changes which take place in graphite after its oxidation and reduction. In order to clarify the description, the product obtained in an oxidation process will be referred as graphite oxide and denoted as $\mathrm{GrO}$ while the product obtained in a reduction process will be referred as graphite oxide and denoted as RGO. The

*E-mail: sabina.drewniak@polsl.pl oxidation process is intended to incorporate the functional groups containing oxygen, e.g. carbonyl, carboxyl, hydroxyl and epoxy between the planes of graphene. The oxidation process was realized using Hummer's method, the reduction process was realized using a temperature of approximately $900^{\circ} \mathrm{C}$ and inert conditions [11]. During the reduction process, the number of additional oxygen and hydrogen groups in $\mathrm{GrO}$ decreases, giving the RGO structure.

The research presented in this paper was conducted using a sample of graphene flakes. Graphite is characterized as an ordered crystallographic structure and has a high content of mineral substance (at 9.7\%).

Preparation of graphite oxide: Commercial flake graphite powder $(90 \mu \mathrm{m})$, supplied by Signograf was oxidized by a modified Hummer method [12]. Concentrated $\mathrm{H}_{2} \mathrm{SO}_{4}$ (95$97 \%$ ) was used as an acid and $\mathrm{KMnO}_{4}$ and $\mathrm{NaNO}_{3}$. A graphite sample $(1 \mathrm{~g})$ with a particle size $<20 \mu \mathrm{m}$ was mixed with the acid and placed in a bath of water and ice. When a homogeneous paste was obtained, the oxidizing agent was partially added, while continuously mixing and maintaining the reaction temperature in the range $10 \div 35^{\circ} \mathrm{C}$. In certain methods, the following amounts of reagents were used: $30 \mathrm{ml}$ of $\mathrm{H}_{2} \mathrm{SO}_{4}, 3 \mathrm{~g} \mathrm{NaNO}$, with $2 \mathrm{~h}$ mixing, the oxidation products were achieved. At the last step, for all methods, the obtained oxidized mixtures were diluted in $100 \mathrm{ml}$ of deionized water (DI), followed by addition of $\mathrm{H}_{2} \mathrm{O}_{2}$ to reduce excess $\mathrm{KMnO}_{4}$ to $\mathrm{MnO}_{2}$. The resulting suspension was centrifuged and the solution was decanted. The prepared precipitate was washed with $10 \%$ $\mathrm{HCI}$, then centrifuged and the resulting slurry was repeatedly washed up with water to obtain $\mathrm{pH} 5$.

Preparation of graphene oxide: High temperature in an inert atmosphere- $\mathrm{N}_{2}$ (temperature $900^{\circ} \mathrm{C}$, approx. $15 \mathrm{~min}$ ) was used to receive reduced graphene oxide from the previously obtained graphite oxide after test expansion.

The resulting products were examined using: electron microscopy, Raman spectroscopy, elementary analyzer and technique using termogravimetry. Figures 1-3 show 
images of graphite, graphite oxide and graphene oxide, respectively (obtained by electron microscopy).

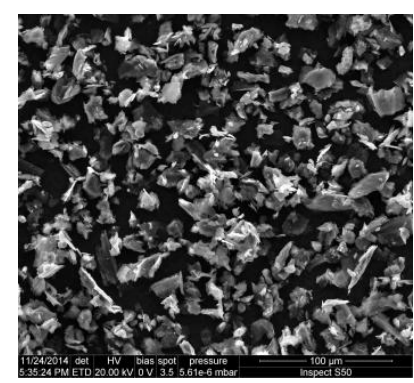

Fig. 1. SEM image of the graphite.

Figure 1 confirms that the size of graphite flakes is less than $20 \mathrm{~nm}$. Figure 2 shows that after the oxidation process of graphite, the size in flakes has not changed significantly, while the image Figure 3 presents the structure of a completely different nature; particles of reduced graphene oxide are much smaller and have a tendency to combine to a form of agglomerates ("cloud structure").

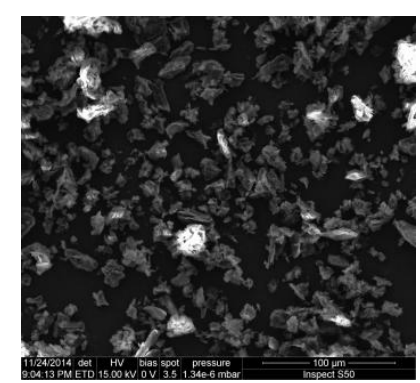

Fig. 2. SEM image of the graphite oxide.

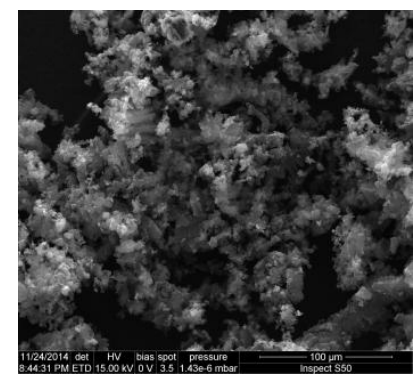

Fig. 3. SEM image of the graphene oxide.

In order to expand the knowledge of the studied structures, thermogravimetric analysis of GrO and RGO was performed. Analyzing the results, it can be seen that the loss in weight occurred in both the oxidized and reduced graphite, during the exposure to a high temperature. The loss of weight was steady in RGO in a wide range of temperatures; only above $900^{\circ} \mathrm{C}$ the process was slightly faster. For the RGO sample, the total weight loss is approx. 21\%. Oxidized graphite performs differently. A sudden loss of weight takes place up to approx. $250^{\circ} \mathrm{C}$ (up to this temperature, the loss of weight is $26 \%$ ). Above this temperature, the changes are slower. The total weight loss of $\mathrm{GrO}$ is $43 \%$.

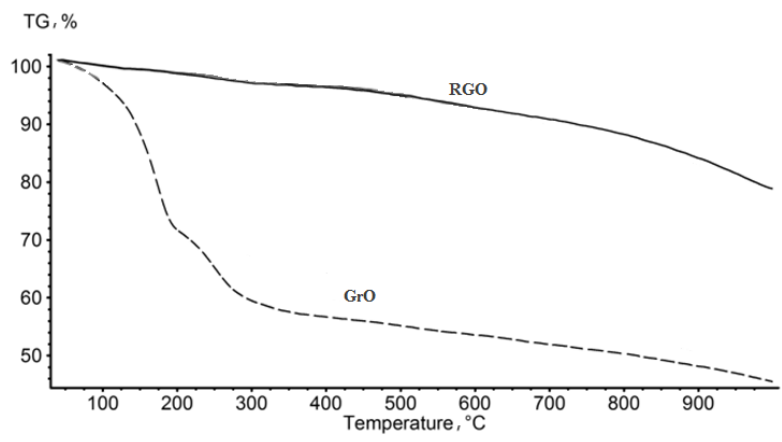

Fig. 4. Thermogravimetric curves for GrO and RGO.

Besides changes in the morphology and topography of graphite, graphite oxide and graphene oxide, the percentages quantities of carbon, hydrogen and oxygen were controlled. At the beginning, the graphite consisted of approx. $\mathrm{C}^{\text {daf }} 99.1 \%$, while the quantity of hydrogen and oxygen was vestigial. The quantity of hydrogen and oxygen in the graphite oxide equaled $2.9 \%$ and $43.5 \%$ respectively, which confirms the connection of many hydroxyl and oxygen groups to the graphite structure. The final percentage quantity of carbon in the reduced product (RGO) amounted to approx. $85 \%$ while the quantity of hydrogen and oxygen was significantly reduced. Table 1 shows changes in the quantities of the above mentioned elements between *) the graphite and graphite oxide and **) graphite oxide and graphene oxide ("+" sign means an increase in the quantity of an elements, "-" the decrease).

Table 1:

\begin{tabular}{|c|c|c|}
\hline & $\begin{array}{c}* \text { ) The difference in } \\
\text { the quantity of each element } \\
\text { of } \\
\text { Gr and GrO, } \%\end{array}$ & $\begin{array}{c}* * \text { ) The difference in } \\
\text { the quantity of each } \\
\text { element of } \\
\text { GrO and RGO, } \%\end{array}$ \\
\hline $\mathrm{C}$ & -46.4 & +42.0 \\
\hline $\mathrm{H}$ & +2.8 & -2.1 \\
\hline $\mathrm{O}$ & +43.2 & -33.9 \\
\hline
\end{tabular}

Raman spectroscopy was the last technique used in our experiments. Raman spectra have been performed for graphite, graphite oxide and graphene oxide (Fig. 5). In the spectrum obtained for graphite, three characteristic peaks are shown (around the wave number equal to $\sim 1350 \mathrm{~cm}^{-1}, 1580 \mathrm{~cm}^{-1}$ and $2700 \mathrm{~cm}^{-1}$. These peaks are the results of disorder in the crystallographic structure of carbon, the vibration of carbon atoms in the plane of layers and number of layers of carbon [13-15]. Based on 
the obtained spectrum, it can be concluded that the graphite structure is to some extent defected and consists of a few/several layers of carbon. Both in the case of graphite oxide and graphene oxide, the first two peaks clearly increased and partly overlapped due to the conducted processes. In the case of graphite oxide, a lower ratio of amplitude of the first peak and second one suggests that the number of defects in the carbon structure increased (attachment of additional functional groups damaged a part of the existing bonds) while in the graphene oxide layers, the same ratio is much higher, which suggests significant defection of the layer. This defection is the consequence of detachment of a large quantity of functional groups.

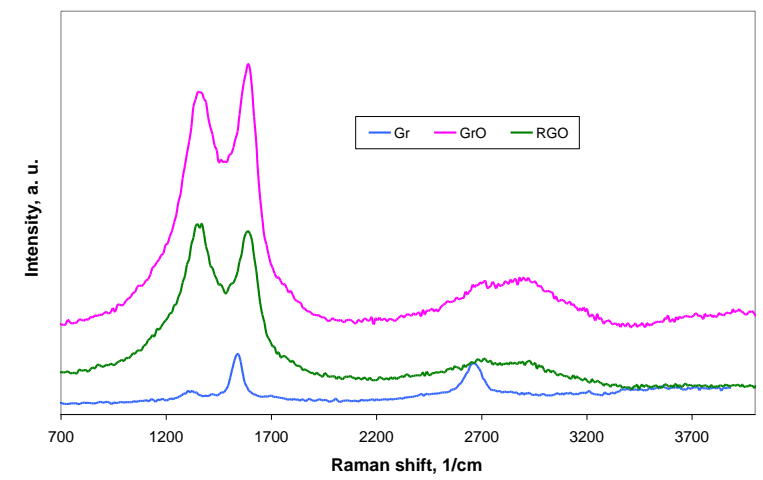

Fig. 5. Raman spectrum of graphite (Gr), graphite oxide ( $\mathrm{GrO})$ and reduced graphite oxide (RGO).

In this paper, the changes in graphite (caused by the oxidation and reduction processes) are described. Measurements were performed, which confirm changes in the quantity of carbon, oxygen and hydrogen in graphite, GrO and RGO. With oxidation of graphite, crystallographic graphite lattice structures changed, which gave (in turn) the possibility of obtaining more defected structures (RGO). The resulting material can be used in sensors technology because many of the bonds (affected by bonds damage) can attach the analyzed molecules to the structure. The absorption of some molecules may cause changes in the optical properties of a sensing layer. A relatively simple and inexpensive RGO fabrication process is an unquestionable advantage of such sensing material, but (expected) poor repeatability of the optical gas sensors reduces the possibilities of its commercialization (however, RGO can be successfully used in different kinds of the sensors).

The work was partially supported by

- the Polish National Science Centre "NCN" within the grant 2012/07/B/ST7/01 471;

- the Statutory R\&D Project IChPW, no 11.14.024.

\section{References}

[1] U. Lange, T. Hirsch, V. M. Mirsky, O.S. Wolfbeis, Electrochimica Acta 562707 (2011).

[2] B.H. Chu, C.F. Lo, J. Nicolosi, C.Y. Chang, V. Chen, W. Strupiński, S.J. Pearton, F. Ren, Sensors and Actuators B 157500 (2011).

[3] L. Ganhua, E.O. Leonidas, C. Junhong, App. Phys. Lett. 94083111 (2009).

[4] C. Botas, P. Alvarez, C. Blanco, R. Santamaria, M. Granda, P. Ares, F. Rodriguez-Reinoso, R. Menendez, Carbon 50, 257 (2012).

[5] L. Sun, B. Fugetsu, Materials Letters 109, 207 (2013).

[6] D. Goers, M.S. Spahr, A. Leone, W. Markle, P. Novak, Electrochimica Acta 563799 (2011).

[7] J.I. Paredes, S. Villar-rodil, M.J. Fernández-Merino, L. Guardia, A. Martínez-Alonso, J.M.D. Tascón, J. Mat. Chem. 21, 298 (2011).

[8] M.H. Tran, C.S. Yang, S. Yang, I J. Kim, H.K. Jeong. Current Applied Phys. 14, 74 (2013)

[9] T. Pustelny, M. Procek, E. Maciak, A. Stolarczyl, S. Drewniak, M. Urbańczyk, M. Setkiewicz, K. Gut, Z. Opilski, Bull. Pol. Ac.: Tech. 64, 4 (2012).

[10] T. Pustelny, M. Setkiewicz, S. Drewniak, E. Maciak, A. Stolarczyk, M. Procek, M. Urbańczk, K. Gut, Z. Opilski, I. Pasternak, W. Strupiński, Acta Phys. Pol. A 122, 5 (2012).

[11] Ł. Smędowski, R. Muzyka, Karbo 2, 128 (2013) in Polish.

[12] Patent US 2798878.

[13] B. Kwiecińska, H. Petersen, Intern. J. of Coal Geology 57, 99 (2004).

[14] Z.Q. Li, C.J. Lu, Z. P. Xia, Y. Zhou, Z. Luo, Carbon 45, 1686 (2007).

[15] Z. Gao, H. Jin, X. Li, Z. Hua. Chem. Res. Chinese 19, 216 (2003). 